\title{
CARTAS AL EDITOR
}

\section{La prevalencia de infección por Chlamydia trachomatis, probablemente mayor a la informada, opinan}

Señor editor: con relación al artículo del doctor Cosme Alvarado Esquivel y colaboradores sobre la Prevalencia de la infección por Chlamydia trachomatis en prostitutas registradas de la ciudad de Durango, publicado en el número de enero-febrero de 2000, considero conveniente realizar algunos comentarios.

La prevalencia de infección por $C$. trachomatis encontrada en esta población $(16.6 \%)$ es elevada tomando en cuenta dos factores: la sensibilidad relativamente baja de la prueba diagnóstica utilizada $(85.4 \%)^{1} y$, sobre todo, debido a que se estudió a un grupo de trabajadoras sexuales a quienes se les practica regularmente exámenes para la detección de infecciones de transmisión sexual (49\%). Por ello, podemos suponer que la prevalencia real de infección por $C$. trachomatis en la población de trabajadoras sexuales de la ciudad de Durango sea mayor a la informada y probablemente similar a la encontrada en la ciudad de México $(20 \%){ }^{2}$

Dos hallazgos interesantes son: la fuerte relación encontrada entre las mujeres con actividad sexual fuera del estado de Durango y la prevalencia de infección por $C$. trachomatis, y la relación inversa entre menor tiempo de práctica de la prostitución y mayor prevalencia de tal infección. En el primer caso, la explicación más próxima sería que la infección por este agente está siendo importada de otros estados y, para el segundo hallazgo, existe, de acuerdo con la literatura internacional, una fuerte asociación de infecciones clamidiales y gonocócicas hasta de $80 \%,{ }^{3}$ ¿será que fueron tratadas con anterioridad por gonorrea y sindromáticamente para clamidia en las otras entidades federativas en donde ejercieron el comercio sexual? $Y$, por ello, esa relación inversa que se encontró, aunado a que el uso del condón, es una práctica elevada de esta población $(98.8 \%)$. La frecuencia encontrada de uso de condón puede constituir un factor de confusión en la interpretación de estos dos resultados. Dos factores pueden ser identificados como causa de esta sobrestimación de uso de condón: a) no se trató de una encuesta anónima y confidencial, y b) el cuestionario fue aplicado por personal médico de la Dirección Municipal de Salud Pública y Medio Ambiente, responsable de otorgar el permiso sanitario, por lo que es probable que las entrevistadas notificaran un mayor uso del condón de lo que es en su práctica real. Otra explicación a estos dos hallazgos sería que el uso de condón probablemente es menor en las trabajadoras sexuales que ejercen fuera del estado de Durango y entre las de menor edad o menor tiempo de ejercer la prostitución como ha sido notificado en otros estudios. ${ }^{4,5}$

Para superar estos detalles metodológicos, los autores debieron aplicar encuestas que garantizaran el anonimato y la confidencialidad de la población estudiada, y para determinar una prevalencia más exacta de la infección por C. trachomatis en esta población era necesario un periodo de estudio mayor que lograra incluir a la subpoblación que acude en forma irregular al examen médico.

Dra. Griselda Hernández Tepichin, Directora Técnica, Consejo Nacional para la Prevención y Control del SIDA, México.

\section{RefERENCIAS}

I. Alvarado-Esquivel C, García-Villanueva A, Castruita-Limones DE, Cardosa-Nevárez FJ, RuizAstorga R. Prevalencia de infección por Chlamydia trachomatis en prostitutas registradas de la ciudad de Durango, México. Salud Publica Mex 2000;42: 43-47.

2. Vázquez $\mathrm{A}$, Cruz C, Vázquez R. Prevalencia de Chlamydia trachomatis en población con prácticas de riesgo, un estudio de 3 años (resumen). Enferm Infecc Microbiol 1998; I8:S40.

3. Gunn RA, Fitzgerald S, Aral SO. Sexually transmitted disease clinic clients at risk for subsequent gonorrhea and Chlamydia infections: Possible "core" transmitters. Sex Transm Dis 2000; 27(6):343-349.

4. Ward H, Day S, Weber J. Risky business: Health and safety in the sex industry over a 9 years period. Sex Transm Infect 1999;75(5):340-343.

5. Fennema JS, van Ameijden EJ, Coutinho RA, van den HoekA. Clinical sexually transmitted diseases among human immunodeficiency virus-infected and noninfected drug-using prostitutes.Associated factors and interpretation of trends, I 986 to 1994. Sex Transm Dis 1997;24(6):363-37I. 


\section{Respuesta de los autores}

Señor editor: agradezco sinceramente y me complace aprender de los amables comentarios de la doctora Griselda Hernández Tepichin, sobre el artículo publicado en Salud Publica Mex 2000; 42(1):43-47.

Ciertamente, los resultados de nuestro trabajo, al utilizar la prueba inmunoenzimática que tiene una sensibilidad de $85.4 \%$, reflejan una prevalencia de infección por Chlamydia trachomatis de $16.6 \%$ en prostitutas registradas de la ciudad de Durango, que pudiera ser más alta si se aplicaran otros métodos con mayor sensibilidad, aunque no tan accesibles desde el punto de vista financiero y de infraestructura. ${ }^{1,2}$ Sin embargo, si los otros métodos alcanzaran $100 \%$ de sensibilidad, incrementarían la prevalencia a $19 \%$ aproximadamente, lo cual, con fines prácticos, no cambia significativamente nuestro mensaje ni la planeación de estrategias que conduzcan al control de la infección.

En cuanto al comentario sobre la posibilidad de una sobrestimación del uso del condón debido a que la encuesta no fue anónima y confidencial, y porque fueron realizadas por el personal médico que otorga el permiso sanitario, deseo aclarar que las entrevistas sí fueron confidenciales. Reconozco, por supuesto, las ventajas de realizar cuestionarios anónimos. Es claro su valor, por ejemplo, en estudios donde los resultados son muy inquietantes para la población analizada, como en aquellos sobre la prevalencia de infección por el virus de inmunodeficiencia humana, y también permiten atraer más gente para algún estudio. ${ }^{3}$ Sin embargo, en nuestra investigación no existían esos problemas. Además, las encuestas anónimas no garantizan respuestas más veraces que aquellas obtenidas en las entrevistas. ${ }^{3,4}$ Las entrevistas tienen también ventajas; los entrevistadores pueden proporcionar más detalles sobre el sentido de alguna pregunta o aclarar alguna duda, y orientar mayormente sobre la manera de contestar e, incluso, los entrevistadores tienen la oportunidad de estimar la veracidad de la respuesta obtenida y volver a preguntar en caso de obtener alguna respuesta poco probable. El no uso de condón no es motivo suficiente para negar un permiso sanitario, asimismo, es creíble que la gran mayoría de las prostitutas y sus clientes lo usen como precaución para evitar contagios. Además, una buena relación entre el médico y la persona entrevistada puede favorecer la obtención de respuestas veraces en la entrevista. Todo en conjunto da como resultado un cuestionario debidamente contestado y confiable.

Respecto al comentario sobre la obtención de una prevalencia más exacta de la infección por $C$. trachomatis en la población estudiada mediante un periodo mayor de estudio que lograra incluir a la subpoblación que acude en forma irregular al examen médico, pienso que la prevalencia obtenida es suficientemente exacta, ya que la población de estudio está claramente definida. Es decir, se especifica que el estudio se realizó únicamente en prostitutas registradas regulares. Ciertamente, a mayor tiempo de estudio, mayor posibilidad de captar prostitutas registradas irregulares, pero no fue el objetivo estudiar una población inconsistente. Nuestro interés fue evaluar únicamente prostitutas registradas regulares porque ellas están activas en la ciudad de Durango y, en consecuencia, representan también el mayor riesgo de diseminación de la infección. Mientras que las prostitutas irregulares, aunque alguna vez se registraron, no tienen permiso sanitario válido, por lo tanto, no ejercen regularmente en la ciudad de Durango. Definitivamente, el factor migración resultó importante en el trabajo, y se recomienda explorarlo adicionalmente en futuros estudios.

Dr. Cosme Alvarado Esquivel, Jefe del Departamento de Inmunología y Biología Molecular, Instituto de Investigación Científica, Universidad Juárez del Estado de Durango, México.

\section{RefERENCIAS}

I. Leyva MA, Rodríguez MA, Martínez NE, Alarcón LC, Flores E. Chlamydia trachomatis en sexoservidoras del estado de Guerrero (resumen). XXII Congreso Nacional de Química Clínica 1999;MSTL 2I.5.

2. Uribe F, Hernández M, Conde C, Juárez L,Allen $B$,Anaya $R$ et al. Low prevalences of $H I V$ infection and sexually transmitted disease among female commercial sex workers in Mexico City. Am J Public Health 1997;87:1012-1015.

3. Goldberg D, Green ST, Taylor A, Frischer M, McKeganey N. Comparison of four survey methods designed to estimate the prevalence of HIV among female prostitutes who inject drugs. Int J STD AIDS 1994;5: | 86- 188.

4. Fehrs LJ, Fleming D, Foster LR, McAlister RO, Fox $V$, Modesitt $S$ et al. Trial of anonymous versus confidential human immunodeficiency virus testing. Lancet 1988;2:379-382.

\section{Discuten aspectos conceptuales y metodológicos de un artículo sobre riesgo nutricional}

Señor editor: enviamos comentarios sobre el artículo Riesgo nutricional en pacientes con tuberculosis pulmonar: ¿cuestión del paciente o de los servicios de salud?, para su consideración por parte de los autores. En dicho artículo éstos llevan a cabo un estudio transversal con el fin de identificar factores de riesgo nutricional en pacientes con tuberculosis pulmonar.

Sin desmérito del estudio realizado, quisiéramos señalar algunas aparentes incorrecciones conceptuales, analíticas y metodológicas presentes en dicho trabajo:

1. La presentación estadística descriptiva de algunos datos no es adecuada. Por ejemplo, la duración media de la tos fue de 3.6 DE \pm 8.6 meses, lo que equivale a decir que aproximadamente $68 \%$ de los valores de duración de la tos se encontraban entre -5 y 12.2 meses. El mismo problema se presenta con los tiem- 
pos de utilización de los antifímicos rifampicina, isoniacida, etambutol y pirazinamida (por ejemplo, el promedio del uso de rifampicina fue de 6.8 y $68 \%$ de los tiempos estuvo entre -5 y 18.6 meses). La distribución tan sesgada de los datos ameritaba la presentación de medianas y cuartiles.

2. El análisis bivariado mostrado en el cuadro III tiene varios errores:

a. En primer lugar, los autores refieren haber calculado razones de prevalencias (RP), lo que no fue así, ya que en realidad obtuvieron razones de momios (RM). Como es sabido, la razón de momios aproxima a la razón de prevalencias sólo si la prevalencia del evento o enfermedad es baja (menor a 20\%). En este caso, la prevalencia de desnutrición cambia de hipótesis a hipótesis, sobre todo debido a la pérdida de datos.

b. Los autores muestran una RP de 0.31 para el "factor de riesgo" gasto de 50\% o más del ingreso familiar en alimentación (que en realidad no resultó de "riesgo" sino de "protección"). En realidad este valor corresponde a una razón de momios, ya que la RP es de 0.67; IC 95\% 0.53 - 0.86. Esto es, el gasto de $50 \%$ o más del ingreso familiar en alimentación disminuye en 33\% (y no en $69 \%$ como indica la razón de momios) la posibilidad de ocurrencia de desnutrición. (Las cursivas son de los autores de la carta).

c. Para la variable atribución de la enfermedad a tipo de alimentación, el estimador puntual de 0.30 es incorrecto, ya que, según las categorías de referencia usadas, la $\mathrm{RM}=3.33$, IC 95\% $0.78-14.15 \mathrm{y}$ $\mathrm{RP}=2.5$, IC 95\% 0.85 - 7.31, ambas con una $p$ de 0.09 . Según el estimador de 0.30 obtenido por los autores, la no atribución de la tuberculosis al tipo de alimentación disminuye en $70 \%$ la posibilidad de desnutrición, cuando en realidad la no atribución de la tuberculosis al tipo de alimentación aumentó 2.5 veces más la posibilidad de ocurrencia de la desnutrición $(R P=2.5)$, independientemente de que sea o no estadísticamente significativo con un alfa de 0.05 .

d. La no utilización del servicio de nutrición se relacionó, según los autores, con una disminución de $58 \%$ en la posibilidad de ocurrencia de desnutrición ( $\mathrm{RP}=$ $0.42)$, es decir, que no usar el servicio de nutrición "protege" contra la desnutrición. De acuerdo con los datos presentados en el cuadro III, la prevalencia de desnutrición fue dos veces mayor entre los que no utilizaron el servicio de nutrición que entre los que sí lo hicieron $(\mathrm{RP}=2$, IC $95 \% 0.58$ - 6.8; $\mathrm{RM}=2.4$, IC $95 \% 0.5-11.5 ; p=0.26$ para ambos estimadores). Dicho de manera recíproca, la utilización del servicio de nutrición se relacionaría con $50 \%$ menos posibilidad de ocurrencia de desnutrición, aunque esto no sea estadísticamente significativo con un alfa de 0.05 .

e. Para la cuarta variable, efectos secundarios a antifímicos, la RP es de 1.17, IC 95\% 0.89 - 1.55, y no de 1.45 .

3. Respecto al modelo de regresión logística múltiple, los autores dan demasiada importancia a la significancia estadística y descuidan el uso de las variables. Por ejemplo, ocupación es una variable nominal que no se debe analizar como ordinal con regresión logística. En este caso la razón de momios, erróneamente llamada razón de prevalencia, nos muestra que por cada unidad de aumento en la ocupación, la posibilidad de desnutrición dis- minuye en promedio $11 \%$, ajustando por las demás variables. Por otra parte, el estimador puntual de la variable atribución de la enfermedad al tipo de alimentación (0.11) no está incluido en el intervalo de confianza (0.72 - 1.27), lo cual se debe tratar de un error tipográfico, al igual que el valor de $\chi^{2}$ de 1058 en el pie de cuadro. Además, si como lo muestra el cuadro III, sólo había disponibles datos de 42 pacientes para analizar la atribución de la enfermedad al tipo de alimentación, al incluir esta variable y otras con valores perdidos, el modelo hará el cálculo con base en el número de datos disponibles comunes a todas las variables. La pérdida de datos arruina el análisis, sobre todo si no se sabe si los datos perdidos difieren de los no perdidos en cuanto a posibles variables confusoras.

4. No se mencionan las categorías de referencia de las variables del modelo; esto es, ¿cuál de los sexos se relacionó con 2.1 mayor posibilidad de desnutrición?, ¿los valores de edad son grupos o años?, ¿cuáles son las unidades de tiempo de evolución? (el estimador de 0.84 muestra que por cada aumento en unidad de tiempo de evolución, la posibilidad de desnutrición disminuye en promedio $16 \%$, lo que no parece razonable, independientemente de la significancia estadística).

5. Los autores se apoyan demasiado en el criterio de significancia estadística. Cabe recordar que el tamaño de muestra se calculó con base en una hipótesis de detección de una prevalencia de $40 \%$; esto es, ese tamaño de muestra es solamente para probar dicha hipótesis. La falta de significancia estadística de las demás hipótesis estudiadas sólo indica que el tamaño de muestra no es suficiente para rechazar cada una de ellas, considerando además que se perdieron datos y se analizaron múltiples hi- 
pótesis, con lo que disminuye el valor de alfa originalmente establecido (0.05).

6. En presencia de los problemas analíticos y conceptuales señalados, la bondad de ajuste del modelo no es evaluable. La conclusión de que los efectos secundarios a antifímicos son "factor de riesgo nutricional" no es precisa, sino más bien un claro ejemplo de causalidad inversa, ya que como los autores lo mencionan "la relación entre desnutrición y TBP es interactiva porque la desnutrición prolongada expone al organismo a una invasión fácil de enfermedades infectocontagiosas y la infección por TBP conduce o agrava la desnutrición".

7. Por último, sugerimos se evite el uso del término "tasa de prevalencia" que aparece en el artículo. Hacemos nuestra la siguiente cita de un texto reciente: "el término tasa de prevalencia se utiliza con frecuencia en lugar de prevalencia. Esto no es correcto ya que la prevalencia, por definición, es una proporción y no una tasa".

Solicitamos encarecidamente a los autores considerar y contestar nuestros comentarios, aclarando que la minuciosidad de los mismos es producto de un análisis grupal realizado como parte de las actividades de aprendizaje del grupo de Epidemiología II, de la Maestría en Salud Pública con área de concentración en Epidemiología.

M. en C. Eduardo Velasco,
Investigador Asociado A,
Instituto Nacional de Salud Pública (INSP), México.
M. en C.Aurelio Cruz Valdez,
defe del Departamento de Epidemiología
M. en C. Ahideé Leyva López,
Investigador Asociado B, INSP, México.
Alumnos: M.C. Javier Reyes Muñoz,
M.C. Carlos Honorato Vázquez,
M.C. Marisol Sánchez Vargas,
Escuela de Salud Pública de México, INSP, México.

\section{Referencias}

I. Núñez-Rocha GM, Salinas-Martínez AM, Villarreal-Ríos E, Garza-Elizondo ME, GonzálezRodríguez F. Riesgo nutricional en pacientes con tuberculosis pulmonar: ¿cuestión del paciente o de los servicios de salud? Salud Publica Mex 2000; 42:126-132.

2. Dos Santos-Silva I. Epidemiología del cáncer: principios y métodos. Lyon (Francia): Organización Mundial de la Salud. Agencia Internacional de Investigación sobre el Cáncer, 1999:63.

\section{Respuesta de los autores a comentarios del grupo de Epidemiología II}

Estimados maestros Eduardo Velasco, Aurelio Cruz y Ahideé Leyva López, y grupo de alumnos de la cátedra de Epidemiología II de la Maestría en Salud Pública con área de concentración en Epidemiología, que participaron en el análisis del artículo: Riesgo nutricional en pacientes con tuberculosis pulmonar: ¿cuestión del paciente o de los servicios de salud? Es para nosotros un honor poder contribuir a la discusión académica y formación de personal en esta área de investigación y, ubicados en ese contexto, comentar lo siguiente en relación con las observaciones que nos hacen.

1. Cuando señalan que variables como la tos y el tiempo de utilización de medicamentos presentaron desviaciones estándar mayores que la media tienen toda la razón, así está documentado en el artículo, también cuando indican que $68 \%$ de la población estudiada se encuentra dentro de la primera desviación estándar, tambien así está señalado, pero tienen un grave problema de interpretación cuando puntualizan que esto equivale a decir que el tiempo de evolución de la tos se encuentra entre -5 y 12.2 meses y que el tiempo de utilización de las drogas antifímicas se encuentra entre -5 y 18.6 meses. Esta interpre- tación la hace el hombre profano, el investigador debe saber que las variables de ese tipo no se comportan con valores negativos, más aún, siendo médico se tiene la obligación de saber que existen algunos valores extremos que están haciendo que el valor de la desviación estándar se comporte de esa manera y que, tal vez, lo más adecuado hubiera sido eliminarlos, pero esto queda a criterio del investigador, a la par se debe tener en cuenta que probablemente una buena cantidad de pacientes cursan con una corta evolución, por lo que es más conveniente presentar la mediana como lo sugieren, pero es pertinente aclarar que este dato lo pueden encontrar en el tercer párrafo de la página 128.

2. Con relación al análisis bivariado contenido en el cuadro III.

a. Tienen razón, las estimaciones corresponden a razón de momios (RM).

b. El valor de 0.31 corresponde a la RM del gasto de $50 \%$ o más del ingreso familiar en alimentación, es decir, es un factor protector para presentar desnutrición, en ninguna parte del documento se dice lo contrario, $y$ en el cuadro se encuentra explícito cuál es el valor de referencia.

c. En la variable "atribución de la enfermedad al tipo de alimentación" sufren una pequeña confusión. Es exactamente lo mismo decir $\mathrm{RM}=3.33$, IC 95\% $0.784-14.15, p=0.09$, que $\mathrm{RM}=$ 0.30 , IC $95 \% 0.06-1.57, p=0.19$, la interpretación va de acuerdo con el enfoque en el análisis de la variable, en una es factor de riesgo y en la otra es factor protector; es importante hacer conciente lo anterior en el grupo de alumnos que están formando. 
En este caso, lo más conveniente hubiera sido que los valores $0.30,0.06-1.57$ y $p=0.19$ se anotaran en el renglón inmediato superior, estamos seguros que esto hubiera evitado su desconcierto.

Creemos que este es un buen ejercicio académico el cual nunca se debe dejar de lado, pero consideramos importante destacar que los valores no reportaron significancia estadística, lo que para fines operativos pierde utilidad y decimos lo anterior recordando que el título del artículo es "Riesgo nutricional en pacientes con tuberculosis pulmonar: ¿cuestión del paciente o de los servicios de salud?" $Y$ en el entendido que para cualquier trabajo de investigación lo más importante, después de su publicación en una revista científica, es la utilización de los resultados por parte de los sistemas de salud, en este caso es evidente que con el resultado arrojado por esta variable en particular no se pueden recomendar acciones concretas a los prestadores de servicio.

d. Nos parece muy grave que señalen que la prevalencia de desnutrición fue dos veces mayor entre los que utilizaron el servicio de nutrición que entre los que no lo hicieron. El cuadro es muy claro, pacientes que utilizaron el servicio de nutrición sin presentar desnutrición: 24; pacientes que no usaron el servicio de nutrición y no presentaron desnutrición: 10; pacientes que usaron el servicio de nutrición y presentaron desnutrición: 4, y pacientes que no utilizaron el servicio de nutrición y presentaron desnutrición: 4 . Francamente, no entendemos la interpretación que se hace.

e. En la variable efectos secundarios a drogas antifímicas, el va- lor de 1.45 , como lo señalaron desde el inicio, corresponde a la RM de prevalencia y no a razón de prevalencia, ya en líneas anteriores se comentó.

3. La operacionalización de la variable ocupación no es la óptima para propiciar y facilitar el análisis. Efectivamente, tienen razón al señalar que existen errores tipográficos (responsabilidad de los autores): en el límite inferior del intervalo de confianza de la variable atribución de la enfermedad al tipo de alimentación se encuentra ausente el signo negativo (-0.72 a 1.27) y en el valor de $\chi^{2}$ del modelo que es de 10.58 faltó el punto, como se establece en el texto en la sección de resultados. Con relación a la pérdida de unidades de observación, más que señalar que arruina el análisis, creemos que la observación se debe enfocar en estimar el nivel de confianza con el que se trabaja, considerando el nuevo tamaño de la muestra.

4. En cuanto a la necesidad de mencionar las categorías de referencia de las variables es conveniente recomandarles la lectura completa del artículo y podrán encontrar en el cuadro I, de la página 129 , la información que solicitan incluyendo la edad.

5. Es correcto. Se estimó el tamaño de la muestra con base en la hipótesis de que la prevalencia de desnutrición en pacientes con tuberculosis pulmonar es de $40 \%$; esto representó un número mínimo de muestra de 125 pacientes. No es del todo correcto señalar que ese tamaño de muestra sea útil solamente para probar dichas hipótesis. Si de manera independiente se estiman tamaños de muestra para cada una de las hipótesis y el número de observaciones mínimas requeridas resulta inferior a las utilizadas en el estudio, entonces el tamaño de muestra utilizado es suficiente para probar el resto de las hipótesis. Una de las posibles razones por las que se presenta la falta de significancia estadística de las demás hipótesis estudiadas, como señalan, se puede deber a que se trabajó con un tamaño de muestra insuficiente, pero no existen los elementos necesarios para afirmarlo, a pesar de que el número de observaciones en alguno de los análisis haya disminuido, esa es una posibilidad, pero mientras no se pruebe objetivamente no se puede descartar que es el comportamiento normal o que tal vez sea un tamaño mínimo de muestra pero con nivel de confianza diferente al 95\%, lo cual evidentemente le restaría valor estadístico. Para afirmar que es debido a un tamaño insuficiente de muestra se deberá calcular primero el tamaño mínimo requerido para cada hipótesis.

6. Una observación que en el último de los casos se podría hacer al modelo de regresión múltiple que se incluye en el trabajo se centraría en la cantidad de variables independientes que fueron incluidas, pero conceptualmente el planteamiento realizado es el adecuado.

7. Estamos de acuerdo, es una proporción y no una tasa de prevalencia.

Agradecemos profundamente el interés académico mostrado en el artículo, consideramos que el análisis crítico es fundamental para nuestro quehacer y esperamos que en este mismo sentido reciban nuestros comentarios. Estamos abiertos a la discusión.

M.S.P. Georgina Mayela Núñez Rocha, ${ }^{(I)}$
Dr. en S.P.Ana Maria Salinas Martínez,,
M.C. S.S. Enrique Villarreal Ríos M, ${ }^{(I)}$
Lic. en Enf. Ma Eugenia Garza Elizondo, ${ }^{(I)}$
M.S.P. Francisco González Rodriguez. ${ }^{(2)}$

(I) Unidad Regional de Investigación Epidemiológica y en Servicios de Salud, Instituto Mexicano del Seguro Social, Monterrey, Nuevo León, México.

(2) Departamento de Medicina Preventiva de la Facultad de Medicina de la Universidad Autónoma de Nuevo León, México. 


\section{Posible sesgo de selección del grupo control}

Señor editor: con la presente enviamos comentarios al artículo Situación psicosocial de las adolescentes y toxemia del embarazo, ${ }^{1}$ producto de un análisis realizado por un grupo de profesores y alumnos del curso de Epidemiología II, de la Maestría en Salud Pública con área de concentración en Epidemiología, del Instituto Nacional de Salud Pública. Asimismo, solicitamos respetuosamente a los autores respuesta a dichos comentarios:

1. En la discusión, los autores refieren que "la selección de los grupos en lo referente a las características sociodemográficas y ginecobstétricas permitió su comparabilidad y el control de estas variables, las cuales son potencialmente confusoras." Al respecto, cabe considerar la posibilidad de que exista sesgo de selección de las pacientes controles, ya que la exclusión de aquellas con algún proceso patológico durante el embarazo que hubiera ameritado hospitalización o que dieron a luz productos con apgar menor a ocho (¿también aquellos que murieron?) pudiera ocasionar una baja representación de la exposición (estresores) entre estas pacientes, en comparación con la población fuente y, por lo tanto, una sobrestimación del efecto de los estresores sobre la toxemia. ${ }^{2}$ Uno de los motivos para excluir a estas pacientes controles hubiera sido evitar una mayor probabilidad de hospitalización de las controles con dichas características; sin embargo, este no es el caso, ya que todas ingresaron para trabajo de parto en el hospital. Si el objetivo era evitar la potencial confusión derivada de dichos criterios (por su posible relación con los estresores y con la toxemia), entonces estas restric- ciones se debieron aplicar también a los casos.

2. Creemos que el tiempo de hospitalización se debió haber considerado como potencial confusor y fuente de sesgo de memoria, debido a que las pacientes con toxemia seguramente estuvieron hospitalizadas por mayor tiempo que las controles, sobre todo al aplicarse los factores de exclusión anteriormente mencionados.

3. En el cuadro I del artículo se presentan, en forma porcentual, algunas características sociodemográficas y ginecobstétricas de adolescentes embarazadas. Independientemente de la falta de significancia estadística con un alfa de 0.05 ; las razones de prevalencias muestran que el ser soltera, tener una escolaridad menor de seis años, ser primigesta y ser empleada o estudiante se rela- cionó con una menor posibilidad de padecer toxemia, lo que parecería ser contraintuitivo respecto a la relación entre estresores y toxemia. Claramente, estas variables ameritaban ser analizadas como factores potencialmente confusores de la relación entre fuentes de estrés, fuentes de apoyo y toxemia.

4. El análisis con razones de prevalencias (RP) hubiera sido más apropiado que el realizado con razones de momios (RM). La razón de momios para comparación de las categorías extremas de percepción de estresores sobrestima su efecto sobre la toxemia $(\mathrm{RM}=4.14 ; \mathrm{IC}$ $95 \% 1.07-15.9$, vs. $\mathrm{RP}=2.2$; IC $95 \%$ $1.2-4.04)$.

5. El periodo transcurrido entre la resolución del embarazo y la entrevista fue de 2.0 meses para las integrantes del primer periodo de

\section{Características sociodemográficas de adolescentes embarazadas. Hospital General de la ciudad de Durango, Durango, MÉxico, 1996-1999}

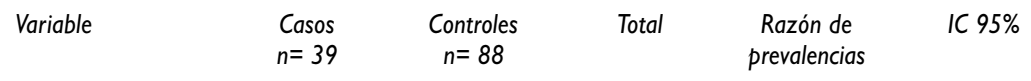

\begin{tabular}{cccccc}
$\begin{array}{c}\text { Estado civil } \\
\text { Soltera }\end{array}$ & 12 & 29 & 41 & 0.93 & $0.52-1.6$ \\
\hline Unida & 27 & 59 & 86 & & \\
$\begin{array}{c}\text { Escolaridad } \\
\leq 6 \text { años }\end{array}$ & 16 & 37 & 53 & 0.97 & $0.57-1.6$ \\
\hline Otros & 23 & 51 & 74 & &
\end{tabular}

\begin{tabular}{lrrrrr}
$\begin{array}{l}\text { Ocupación } \\
\text { Empleada, estudiante }\end{array}$ & 3 & 12 & 15 & 0.62 & $0.22-1.8$ \\
\hline Ama de casa & 36 & 76 & 112 & & \\
Paridad & & & & & \\
$\quad$ Primigestas & 29 & 70 & 99 & 0.82 & $0.45-1.5$ \\
\hline Otros & 10 & 18 & 28 & & \\
$\begin{array}{l}\text { Control prenatal } \\
\text { Sin control }\end{array}$ & 8 & 14 & 22 & 1.23 & $0.65-2.3$ \\
\hline Otros & 31 & 74 & 105 & &
\end{tabular}

Nota: las variables están condensadas, no aparecen como en el original 
recolección de datos y de 4.6 meses para las del segundo, lo cual es una fuente potencial de sesgo de memoria. Este tipo de sesgo puede producir "causalidad inversa", esto es, que la toxemia está relacionada con una mayor percepción y recuerdo de estresores y no viceversa.

Deseamos manifestar a los autores nuestro reconocimiento a su esfuerzo en la realización de este estudio, así como nuestro agradecimiento por producir material que nos ha permitido reflexionar sobre consideraciones metodológicas que quizá no fueron presentadas por cuestiones obvias de espacio.

M. en C. Héctor Eduardo Velasco Mondragón, Investigador Asociado A, Instituto Nacional de Salud Pública (INSP), México.

M. en C. Ahideé Leyva López, Investigadora Asociada B, INSP, México;

Alumnos: M.C. Fernando Briseño Tavez, M.C. Javier Escobar Meza, M.C. Javier Fuentes Domínguez, M.C. Lourdes Fuentes Román,

M.C. Leticia Jiménez Gallegos,

Escuela de Salud Pública de México, INSP, México.

\section{REFERENCIAS}

I Salvador-Moysén J, Martínez-López Y, LechugaQuiñones A, Ruiz-Astorga R, Terrones-González A. Situación psicosocial de las adolescentes y toxemias del embarazo. Salud Publica Mex 2000;42:99-105.

2 Rothman KJ, Greenland S. Modern Epidemiology. 2a. edición Filadelfia: Lippincott-Raven, 1998: 101-102.

\section{Respuesta de los autores}

Señor editor: los autores damos respuesta a los comentarios realizados al artículo Situación psicosocial de las adolescentes y toxemias del embarazo, por un grupo de profesores y alumnos del curso de Epidemiología II, de la Maestría en Salud Pública del Instituto Nacional de Salud Pública.
1. Los criterios de exclusión aplicables al grupo control se establecieron precisamente para asegurar -en la medida de lo posible- la comparabilidad de los grupos; la razón de establecer dichos criterios fue la siguiente: existe una gran cantidad de artículos en los que se plantea la relación de una situación psicosocial desfavorable con la ocurrencia de diferentes eventos patológicos durante la gestación y no únicamente con las toxemias; de tal forma que haber incluido controles con alguna enfermedad durante el embarazo hubiera representado una falla metodológica del diseño por otra parte, sí quisimos establecer la diferencia entre la exposición no sólo a los estresores sino también al apoyo psicosocial, ya que son los elementos que subyacen a la hipótesis del estudio.

2. Las pacientes con preeclampsia leve permanecen hospitalizadas el mismo tiempo que las madres "sanas"; quienes tienen el diagnóstico de preeclampsia severa permanecen hospitalizadas en términos generales 48 horas después de parir, y la estancia de las pacientes con eclampsia, en promedio, es de una semana, no creemos que esta circunstancia represente una fuente de confusión que se haya traducido en un sesgo de memoria.

3. Uno de los aspectos teórico-metodológicos que representó un alto nivel de dificultad para el desarrollo del trabajo, estuvo constituido por la necesidad de definir y medir la situación psicosocial de una manera confiable y con un nivel de exactitud aceptable, estamos convencidos de que el abordaje al evaluar las dimensiones más significativas del contexto de la adolescente embarazada es la que permite obtener información más satisfactoria; existe un gran número de estudios que han analizado el impacto de variables aisladas y los resultados han sido contradictorios. También creemos que el hecho de que haya 1.07 adolescentes sanas solteras por 1.0 adolescente con toxemia no tiene ni significado estadístico ni implicaciones causales; asimismo, el que exista 1.77 adolescentes sanas que son empleadas o estudiantes por cada adolescente con toxemia lo explicamos en función del mayor apoyo que tuvieron las "sanas" para el desarrollo de actividades fuera de su casa, sin que el dato aislado tenga una relación directa con la ocurrencia de la enfermedad.

4. La razón de momios o razón de productos cruzados se utiliza para conocer la fuerza de asociación en estudios de casos y controles, particularmente en estudios que involucran casos incidentes; ${ }^{1}$ mediante este procedimiento se evalúa la relación que existe entre la exposición a un factor de riesgo y la ocurrencia del evento de interés; la lógica que seguimos fue la siguiente: una puntuación de cero o menos significa la presencia del factor de riesgo (situación psicosocial negativa); una puntuación de 5 a 7 (situación psicosocial fuertemente positiva) significa la ausencia del factor de riesgo o, dicho correctamente, la presencia de un factor protector, la misma lógica aplicamos para la evaluación del estado anímico positivo y convivencia satisfactoria.

5. Al considerar la importancia que tiene en la mujer la gestación, no sólo como proceso biológico, sino psicoemocional, es difícil asumir que olvidarán o interpretarán de manera diferente en un periodo de pocos meses el tipo de interacción que establecieron durante su embarazo con las personas que son significativas para ellas. Sin embargo, el argumento más importante se sustenta en la experiencia de los médicos especialistas del Servicio de Ginecobstetricia, quienes opinaron que las entrevistadas 
recordarían con objetividad los aspectos explorados por el instrumento de riesgo psicosocial aplicado.

Agradecemos sinceramente el interés mostrado por nuestro artículo y, efectivamente, existen aspectos no sólo metodológicos sino epistemológicos y conceptuales no discutidos, que ojalá en otro manuscrito podamos abordar.

M. en C. Jaime Salvador Moysén, (I)

M. en C.Yolanda Martínez López, ${ }^{(2)}$

Psic. Angélica Lechuga Quiñones, (I) Q.F.B. Rosario Ruiz Astorga, (I) Psic. Alberto Terrones González. (I)

(I) Instituto de Investigación Cientifica, Universidad Juárez del Estado de Durango. Durango, México.
(2) Unidad de Epidemiología Clínica, Hospital General de Durango, Servicios de Salud de Durango, México.

\section{ReferenCias}

I. Kleinbaum DG, Kupper L, Morgenstern H. Epidemiologic research. Nueva York: Editorial Van Nostrand Reinhold, 1982:141-147. 